\title{
Health Literacy in Glomerulonephritis and Renal Vasculitis Attending Nephrology Clinics
}

\author{
Cynthia Ciwei Lim ${ }^{a}$ Irene Y.J. Mok ${ }^{a}$ Hui Zhuan Tan ${ }^{a}$ Claire Tan ${ }^{a}$ Fiona Yeo ${ }^{b}$ \\ Jason C.J. Choo ${ }^{\mathrm{a}}$ \\ ${ }^{a}$ Department of Renal Medicine, Singapore General Hospital, Singapore, Singapore; ${ }^{b}$ Pharmacy, Singapore General \\ Hospital, Singapore, Singapore
}

\section{Keywords}

Patient education · HLS-EU-Q47

\begin{abstract}
Aims: Glomerulonephritis is one of the leading causes of progressive chronic kidney disease worldwide and treatment requires shared decision-making to improve self-care and plan immunosuppressant therapy. However, information on health literacy $(\mathrm{HL})$ in patients with glomerulonephritis is scanty. We aimed to assess $\mathrm{HL}$ in our multiethnic population with glomerulonephritis. Methods: Single-center cross-sectional study of patients with glomerulonephritis receiving induction immunosuppressants at the ambulatory nephrology clinic and who completed the anonymized selfadministered HLS-EU-47 questionnaire. The standardized HL index and domain item mean scores were compared with participant sociodemographic characteristics. Results: Among 65 patients who attend the clinics over a month, 27 agreed to participate in the survey. After excluding responses with significant missing information, we included 23 participants (16 Chinese, 4 Malay, 2 Indian, and 1 other ethnicity) in the analysis. The median age was 39 (interquartile range 27, 60 years). The median general HL index was 26.2 (19.8,
\end{abstract}

karger@karger.com www.karger.com/gdz

Karger $\stackrel{\text { ' }}{5}$
(C) 2021 The Author(s)

Published by S. Karger AG, Basel

This is an Open Access article licensed under the Creative Commons Attribution-NonCommercial-4.0 International License (CC BY-NC) (http://www.karger.com/Services/OpenAccessLicense), applicable to the online version of the article only. Usage and distribution for commercial purposes requires written permission.
29.8). The item mean scores were $2.64(2.43,2.77), 2.45$ (2.09, $2.72), 2.33(2.17,2.58)$, and $2.50(2.25,2.75)$ for the domains of accessing, understanding, appraising, and applying health-care-related information, respectively. Male patients had significantly higher $\mathrm{HL}$ indices and higher scores for accessing and appraising health information, while higher personal income was significantly associated with higher score for applying health knowledge. Conclusion: Patients with glomerulonephritis had lowest $\mathrm{HL}$ in the domain of appraising health information. Further research on targeted interventions to improve the $\mathrm{HL}$ in appraising treatment options and vaccinations in patients with glomerulonephritis is required.

(c) 2021 The Author(s).

Published by S. Karger AG, Basel

\section{Introduction}

Glomerulonephritis is one of the leading causes of progressive chronic kidney disease worldwide [1,2]. Clinical management entails self-care improvements to retard chronic kidney disease progression and treatment decisions for potent immunosuppressants that can potentially cause severe adverse effects $[3,4]$ and thus involves shared 
decision-making between the physician and patients. This in turn requires patients to understand their disease conditions. Health literacy (HL) is defined by the ability to access, understand, evaluate, and apply health-care-related information and services to make informed health decisions [5]. Low HL is associated with poorer health outcomes [6], possibly because it limits patients' engagement in their care, including adherence to long-term therapy [7]. Knowledge of patients' HL allows for targeted patient education and efficient health promotion and health policy implementation [8]. Indeed, interventions in patients with low HL have been shown to improve patient outcomes [9]. However, information on HL in patients with glomerulonephritis is scanty. A systematic review of HL studies in chronic kidney disease identified 13 studies with 13,202 dialysis patients but only 12 studies, among which 8 were abstracts, involved 4,367 patients with nondialysis kidney disease [6]. None of the publications specifically evaluated patients with glomerulonephritis [10-12].

Furthermore, there is no international consensus regarding an appropriate HL assessment tool. A literature review of $17 \mathrm{HL}$ assessment tools found that the Test of Functional HL in Adults (TOFHLA) and the Rapid Estimate of Adult Literacy in Medicine (REALM) HL Test were most commonly used [13], while the aforementioned review of HL in chronic kidney disease identified the Short Test of Functional HL and REALM as popular choices [6]. TOFHLA is a reading test of 125 medical terms and a subscale to measure numeracy skills, whereas Short Test of Functional HL comprise 36 reading comprehension items, and REALM is scored based on number of correctly pronounced words from a total of 66 words but were criticized for lack of coverage for HL domains such as comprehension of printed health materials, numeracy, and information navigation [14]. Both TOFHLA and REALM are available in English, Spanish, and Portuguese, but there were no validated versions in both Chinese and Malay for the ethnically diverse population in Singapore at the time of study conception. In contrast, the European Health Literacy Survey (HLS-EU-Q47), originally developed and validated in Europe $[15,16]$, was translated into Malay and traditional Chinese and validated in several Asian countries such as Malaysia, Vietnam, and Taiwan [17]. The questionnaire covers the areas of accessing, understanding, evaluating, and applying health-care-related information in the domains of health care, disease prevention, and health promotion $[15,17]$. It was found to have good construct validity (goodness of fit indices $>0.85$ for all domains), satisfactory item-scale convergent validity (item-scale correlation $\geq 0.40$ ), high internal consistency
(Cronbach's alpha $>0.90$ ), and no floor or ceiling effect [17]. We thus aimed to assess HL in our ethnically diverse patients with glomerulonephritis using the HLS-EU-Q47 and its translations in a pilot study.

\section{Methods}

This was a single-center cross-sectional study of patients with glomerulonephritis and renal vasculitis who attended the weekly ambulatory Glomerulonephritis Disease Management clinics in the Singapore General Hospital, an academic medical center and tertiary referral center, over a month. This clinic manages patients with active glomerulonephritis and renal vasculitis who require induction immunosuppressive therapy. Patients were invited to participate in the self-administered questionnaire by clinic staff. We collected and categorized sociodemographic data such as age, gender, ethnicity (Chinese, Malay, Indian, or others), highest education attained (university and above vs. not), occupation (professional or executive vs. not), personal gross monthly income (less than SGD 2,000 vs. not), language spoken at home (English, Mandarin, Malay, Tamil, and others), language used to communicate with health-care workers (English, Mandarin, Malay, Tamil, and others), and frequency of visits to health-care institutions such as clinic or hospital in the last 6 months (4 or more vs. not); as well as comorbid and medication history. The HLS-EU-Q47 is a self-reported 47-item paper-pencil survey originally available in English, traditional Chinese, and Malay [17]. The Asian Health Literacy Survey Consortium approved the conversion of the traditional Chinese version to simplified Chinese, which is the standard writing form taught locally. The questionnaire and the translations are available in online suppl. Table 1a-c; for all online suppl. material, see www.karger.com/doi/10.1159/000517886. Patients were offered the language version of their preference. Each item was rated on a 4-point Likert scale for perceived level of difficulty ( 1 = very difficult, $2=$ difficult, $3=$ easy, and $4=$ very easy). This study was exempted from review and documented informed consent by the SingHealth Institutional Review Board (CIRB 2020/227) since the study involved a survey without identifiers.

\section{Statistical Analysis}

Statistical analysis was performed using IBM SPSS Statistics 26 (IBM Corp., Armonk, NY, USA). Categorical variables were presented as proportions, and continuous variables summarized as medians with interquartile ranges (25th percentile and 75th percentile). The HL index for each patient was standardized based on a formula: index $=($ mean score -1$) \times(50 / 3)$, where mean score was the mean of all participating items that each patient answered, 50 was the chosen maximum value and 3 was the range of possible mean scores [16, 17]. Thus, a HL index value was obtained where 0 and 50 represented the lowest and the highest HL, respectively. We calculated the item mean scores (sum score/number of items with response) of each of the literacy domains of accessing (13 items), understanding (11 items), appraising (12 items), and applying (11 items) health information (online suppl. Table 2) in order to obtain greater insight into perceived difficulties in each domain [18]. We compared the HL index and domain item mean scores according to participant characteristics using the Mann Whitney $U$ test. All analyses were two-tailed and $p$ values $<0.05$ were considered statistically significant. 


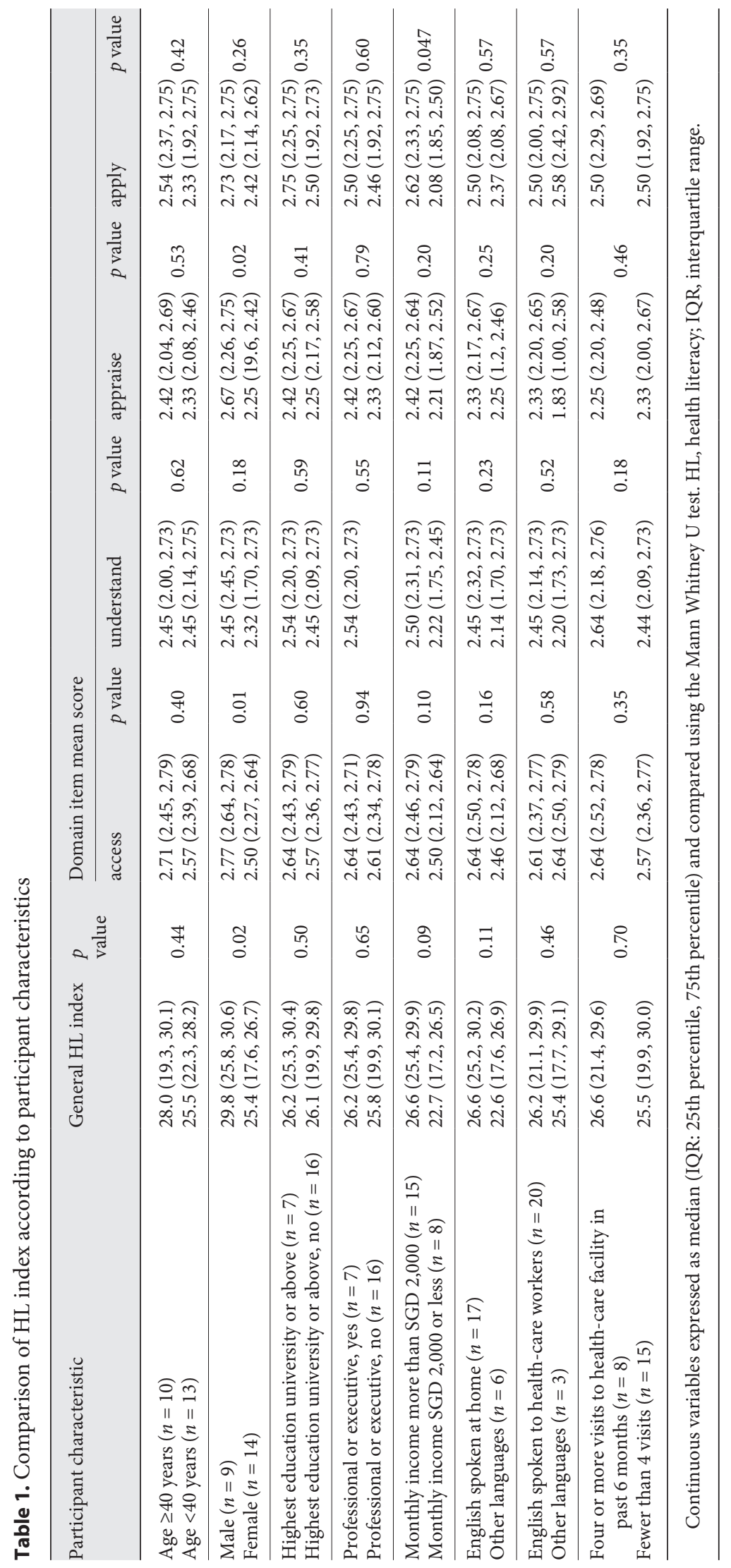




\section{Results}

Among 65 patients who attended the weekly ambulatory Glomerulonephritis Disease Management clinics over a month, 27 agreed to participate in the survey. After excluding responses with missing information for $>10 \%$ of the sociodemographic data ( 2 responses) and $>5 \%$ of the HLS-EU-Q47 questionnaire (2 responses), we included 23 participants (16 Chinese, 4 Malay, 2 Indian, and 1 other ethnicity) in the analysis. The median age was 39 (27, 60 years), and few had comorbidities such as diabetes $(n=2)$ and hypertension $(n=3)$. The most frequent language version of the HLS-EU-47 questionnaire chosen by participants was English $(n=19)$, followed by Chinese $(n$ $=3)$ and Malay $(n=1)$.

The median general HL index was $26.2(19.8,29.8)$. The item mean scores were $2.64(2.43,2.77), 2.45(2.09,2.72)$, $2.33(2.17,2.58)$, and $2.50(2.25,2.75)$ for the domains of accessing, understanding, appraising, and applying health-care-related information, respectively. Online suppl. Table 3 shows the proportion of responses for individual items that were perceived as "difficult" or "very difficult" by participants. The areas with greatest perceived difficulty were related to accessing, appraising, and applying information on different treatment options to make therapeutic decisions, appraising need for vaccinations and health screening, and appraisingand applying information related to illness and health risks in the media.

The HL index and domain item mean scores were compared according to participant characteristics (Table 1). Male patients had significantly higher general HL than female patients with glomerulonephritis. Male gender was also significantly associated with higher item mean scores for accessing and appraising health information. Higher personal income was significantly associated with higher item mean score for applying health knowledge and tended to be associated with higher general HL and higher item mean score for accessing health knowledge.

\section{Discussion}

The general HL index among patients with active glomerulonephritis receiving treatment with immunosuppressants was $26.2(19.8,29.8)$ and higher in males compared to females, while the domain item mean score was lowest for appraising health information. Current knowledge of HL in patients with glomerulonephritis is scanty and meaningful comparisons are made more difficult by the lack of uniform assessment tools. A systematic review on HL in chronic kidney disease did not identify any study published before 2016 that specifically evaluated patients with glomerulonephritis [6]. More recently, a HL study that used the HLS-EU-47 questionnaire to assess 200 predialysis Japanese patients included 42 with chronic glomerulonephritis but did not report the latter's HL as a group [19]. A Canadian single-center cross-sectional study included 20 pre-dialysis patients with glomerulonephritis but similarly did not report their HL [20], although it noted that pre-dialysis patients were less confident in understanding health information compared to those on dialysis. While a Norwegian single-center cross-sectional study included 40 patients with glomerulonephritis, among whom 22 were assessed by the Health Literacy Questionnaire to have mid-level HL [7], these were both pre- and on-dialysis patients and the HL of the pre-dialysis group was not reported separately. A Taiwanese study that included 201 individuals with chronic kidney disease due to glomerulonephritis found that $34.3 \%$ had low scores for both self-care and disease knowledge [21]; only a quarter scored highly for these aspects related to HL.

As clinicians managing patients with glomerulonephritis, it is concerning that the domain item mean score was lowest for appraising health information and nearly a quarter of responders perceived difficulty related to accessing, appraising and applying information on different treatment options to make therapeutic decisions (items 2, 10, and 13). Management of glomerulonephritis often involves discussion regarding the clinical trajectory of the condition and need for potent immunosuppressants to achieve remission, but these medications may be associated with side effects such as infectious and metabolic complications $[3,4$, 22]. Patients involved in the shared decision-making may find weighing the relative benefits and risks of the myriad and complex therapeutic options considerably daunting. We have initiated pharmacist counseling sessions and provided written patient education material to improve access to information regarding immunosuppressants. While we have prepared these materials in English, Chinese, and Malay for our multiethnic population, we noted that most participants spoke English at home and at health-care facilities and the most frequent language version of the HLS-EU-47 questionnaire chosen by our multiethnic participants was English (79.2\%). Additionally, it was interesting that the areas with greatest perceived difficulty (approximately athird of responses) were related to appraising the need for vaccinations and health screening, and appraising and applying information related to illness and health risks in the media to improve health. This survey was conducted in mid-2020 during the first wave of the COVID-19 pandem- 
ic, also termed the pandemic of misinformation [23], and a global race toward vaccine development [24]. Further studies will be required to evaluate if the widespread media coverage of the pandemic and the available vaccines over the past year have resulted in improved HL in these aspects.

This study has several limitations. The small sample size limits the ability to detect significant associations with small effect size and precluded multivariate analysis to assess for possible confounders. The results from this singlecenter study may not be generalizable to cohorts with different educational and socioeconomic profiles or healthcare systems. Despite the limitations, this study attempted to explore the knowledge gap in HL among patients with glomerulonephritis. It assessed the HL among patients with glomerulonephritis on immunosuppressants and identified patients' concerns regarding their ability to appraise health information. The results obtained from this pilot can guide further research and interventions to improve the access, appraisal, and application of health information, especially regarding treatment options and vaccinations, in patients with glomerulonephritis.

\section{Acknowledgements}

The study team thanks Professor Peter Chang and Dr Tin Su $\mathrm{Su}$ of the Asian Health Literacy Survey Consortium for approving the use of the translated versions of HLS-EU-Q47.

\section{Statement of Ethics}

This study was exempted from review and documented informed consent by the SingHealth Centralized Institutional Review Board (CIRB 2020/227) for this survey without the use of identifiers.

\section{Conflict of Interest Statement}

No relevant conflicts of interest for all authors.

\section{Funding Sources}

The authors did not receive any funding.

\section{Author Contributions}

C.L. conceptualized the study and wrote the 1 st draft; C.L., I.M., and H.Z.T. recruited participants; C.T. and C.L. performed data entry and analysis; and all the authors reviewed and approved the manuscript.

\section{Availability of Data and Material}

Dataset available upon reasonable request to the study team, subject to institutional approval, and data sharing agreement.

\section{References}

1 Maisonneuve P, Agodoa L, Gellert R, Stewart $J H$, Buccianti G, Lowenfels AB, et al. Distribution of primary renal diseases leading to endstage renal failure in the United States, Europe, and Australia/New Zealand: results from an international comparative study. Am J Kidney Dis. 2000;35:157-65.

2 Gan L, Zuo L. Current ESRD burden and its future trend in Beijing, China. Clin Nephrol. 2015;83:17-20.

3 Lim CC, Liu PY, Tan HZ, Lee P, Chin YM, Mok IY, et al. Severe infections in patients with lupus nephritis treated with immunosuppressants: a retrospective cohort study. Nephrology. 2017;22(6):478-84.

4 Lim CC, Gardner D, Ng RZ, Chin YM, Tan HZ, Mok IY, et al. Synergistic impact of prediabetes and immunosuppressants on the risk of diabetes mellitus during treatment of glomerulonephritis and renal vasculitis. Kidney Res Clin Pract. 2020;39(2):172.

5 Sorensen K, Van den Broucke S, Fullam J, Doyle G, Pelikan J, Slonska Z, et al. Health literacy and public health: a systematic review and integration of definitions and models. BMC Public Health. 2012;12:80.
6 Taylor DM, Fraser S, Dudley C, Oniscu GC, Tomson C, Ravanan R, et al. Health literacy and patient outcomes in chronic kidney disease: a systematic review. Nephrol Dial Transplant. 2018;33:1545-58.

7 Elisabeth Stømer U, Klopstad Wahl A, Gunnar Gøransson L, Hjorthaug Urstad K. Health literacy in kidney disease: associations with quality of life and adherence. J Renal Care. 2020;46:85-94.

8 Beauchamp A, Batterham RW, Dodson S, Astbury B, Elsworth GR, McPhee C, et al. Systematic development and implementation of interventions to OPtimise Health Literacy and Access (Ophelia). BMC Public Health. 2017;17(1):230.

9 Sheridan SL, Halpern DJ, Viera AJ, Berkman $\mathrm{ND}$, Donahue KE, Crotty K. Interventions for individuals with low health literacy: a systematic review. J Health Commun. 2011;16(Suppl 3):30-54.

10 Devraj R, Borrego M, Vilay AM, Gordon EJ, Pailden J, Horowitz B. Relationship between health literacy and kidney function. Nephrology. 2015;20(5):360-7.

11 Wright JA, Wallston KA, Elasy TA, Ikizler TA, Cavanaugh KL. Development and results of a kidney disease knowledge survey given to patients with CKD. Am J Kidney Dis. 2011; 57(3):387-95.

12 Nunes JAW, Anderson CA, Greene JH, Ikizler TA, Cavanaugh KL. Results of a novel screening tool measuring dietary sodium knowledge in patients with chronic kidney disease. BMC Nephrol. 2015;16:17.

13 Altin SV, Finke I, Kautz-Freimuth S, Stock S. The evolution of health literacy assessment tools: a systematic review. BMC Public Health. 2014;14:1207.

14 Dumenci L, Matsuyama RK, Kuhn L, Perera RA, Siminoff LA. On the Validity of the Rapid Estimate of Adult Literacy in Medicine (REALM) Scale as a Measure of Health Literacy. Commun Methods Meas. 2013;7(2): 134-43.

15 Sorensen K, Van den Broucke S, Pelikan JM, Fullam J, Doyle G, Slonska Z, et al. Measuring health literacy in populations: illuminating the design and development process of the European Health Literacy Survey Questionnaire (HLS-EU-Q). BMC Public Health. 2013; 13:948. 
16 Sorensen K, Pelikan JM, Rothlin F, Ganahl K, Slonska Z, Doyle G, et al. Health literacy in Europe: comparative results of the European health literacy survey (HLS-EU). Eur J Public Health. 2015;25:1053-8.

17 Duong TV, Aringazina A, Baisunova G, Nurjanah, Pham TV, Pham KM, et al. Measuring health literacy in Asia: validation of the HLSEU-Q47 survey tool in six Asian countries. J Epidemiol. 2017;27:80-6.

18 van der Heide I, Rademakers J, Schipper M, Droomers M, Sørensen K, Uiters E. Health literacy of Dutch adults: a cross sectional survey. BMC Public Health. 2013;13:179.
19 Kita Y, Machida S, Shibagaki Y, Sakurada T. Fact-finding survey on health literacy among Japanese predialysis chronic kidney disease patients: a multi-institutional cross-sectional study. Clin Exp Nephrol. 2021;25(3):224-30.

20 Boyer A, Begin Y, Dupont J, Rousseau-Gagnon $\mathrm{M}$, Fernandez N, Demian $\mathrm{M}$, et al. Health literacy level in a various nephrology population from Quebec: predialysis clinic, in-centre hemodialysis and home dialysis; a transversal monocentric observational study. BMC Nephrol. 2021;22(1):259.
21 Tsai YC, Wang SL, Tsai HJ, Chen TH, Kung LF, Hsiao PN, et al. The interaction between self-care behavior and disease knowledge on the decline in renal function in chronic kidney disease. Sci Rep. 2021;11(1):401.

22 Lim CC, Tung YT, Tan BH, Lee PH, Mok I, Oon L, et al. Epidemiology and risk factors for cytomegalovirus infection in glomerular diseases treated with immunosuppressive therapy. Nephrology. 2017;23(7):676-81.

23 Li HO, Bailey A, Huynh D, Chan J. YouTube as a source of information on COVID-19: a pandemic of misinformation? BMJ Glob Health. 2020;5(5):e002604.

24 Mullard A. COVID-19 vaccine development pipeline gears up. Lancet. 2020;395(10239): $1751-2$ 